\title{
Drosophila mitoferrin is essential for male fertility: evidence for a role of mitochondrial iron metabolism during spermatogenesis
}

\author{
Christoph Metzendorf and Maria I Lind*
}

\begin{abstract}
Background: Mammals and Drosophila melanogaster share some striking similarities in spermatogenesis. Mitochondria in spermatids undergo dramatic morphological changes and syncytial spermatids are stripped from their cytoplasm and then individually wrapped by single membranes in an individualization process. In mammalian and fruit fly testis, components of the mitochondrial iron metabolism are expressed, but so far their function during spermatogenesis is unknown. Here we investigate the role of Drosophila mitoferrin (dmfrn), which is a mitochondrial carrier protein with an established role in the mitochondrial iron metabolism, during spermatogenesis.
\end{abstract}

Results: We found that P-element insertions into the 5 '-untranslated region of the dmfrn gene cause recessive male sterility, which was rescued by a fluorescently tagged transgenic $d m f r n$ genomic construct (dmfrnvenus). Testes of mutant homozygous dmfrn ${ }^{S H 115}$ flies were either small with unorganized content or contained some partially elongated spermatids, or testes were of normal size but lacked mature sperm. Testis squashes indicated that spermatid elongation was defective and electron micrographs showed mitochondrial defects in elongated spermatids and indicated failed individualization. Using a LacZ reporter and the $d m f r n v e n u s$ transgene, we found that dmfrn expression in testes was highest in spermatids, coinciding with the stages that showed defects in the mutants. Dmfrn-venus protein accumulated in mitochondrial derivatives of spermatids, where it remained until most of it was stripped off during individualization and disposed of in waste bags. Male sterility in flies with the hypomorph alleles dmfrnBG00456 and dmfrn EY01302 over the deletion Df(3R)ED6277 was increased by dietary iron chelation and suppressed by iron supplementation of the food, while male sterility of $d$ mfrnSH115/Df(3R)ED6277 flies was not affected by food iron levels.

Conclusions: In this work, we show that mutations in the Drosophila mitoferrin gene result in male sterility caused by developmental defects. From the sensitivity of the hypomorph mutants to low food iron levels we conclude that mitochondrial iron is essential for spermatogenesis. This is the first time that a link between the mitochondrial iron metabolism and spermatogenesis has been shown. Furthermore, due to the similar expression patterns of some mitochondrial iron metabolism genes in Drosophila and mammals, it is likely that our results are applicable for mammals as well.

\section{Background}

Iron is an essential micronutrient for almost all organisms and is used as a co-factor for many enzymes involved in redox-reactions. Its reactivity with hydrogenperoxide also bears the potential to promote the formation of reactive oxygen species via the Fenton reaction. Reactive oxygen species in turn result in, protein, lipid

* Correspondence: maria.lind@ebc.uu.se

${ }^{1}$ Comparative Physiology, Uppsala University, Norbyvägen 18A, 75236 Uppsala, Sweden

Full list of author information is available at the end of the article and DNA damage that can lead to cellular dysfunction and damage to organs. Consequently, free iron levels must be kept at a minimum, while enough iron must be provided to processes that depend on it (reviewed in $[1,2])$.

Mitochondria are the sites of iron-insertion into protoporphyrin IX [2] and iron-sulfur cluster (ISC) biosynthesis $[3,4]$ within eukaryotic cells and are, therefore, the subcellular compartments with the highest requirement for iron. Transport of iron into mitochondria, is facili- 
tated by the mitochondrial carrier proteins Mrs3p and Mrs4p (Mrs3/4p) [5,6] in yeast. MRS3/4 genes have been shown to genetically interact with frataxin [7] in the delivery of iron to heme [8] and ISC synthesis [9] in mitochondria. At least in yeast, another less effective mitochondrial iron transport mechanism seems to exist, as $M R S 3 / 4$ mutants only manifest phenotypes at low iron conditions [10].

In vertebrates two paralog genes that are homologs to $M R S 3 / 4$ exist. Mitoferrin1, is mainly expressed in erythropoietic tissues and the frascati mutations in the zebrafish result in hemoglobinization defects, anemia and lethality [11]. Both mitoferrin1 and mitoferrin 2 can rescue yeast $M R S 3 / 4$ double mutants, indicating a similar function. Only ectopic expression of mitoferrin 1 can rescue frascati mutants [11] and it was recently shown that mitoferin1 protein, but not mitoferrin2, accumulates in erythropoietic cells at amounts that can meet the need of mitochondria for iron in these cells [12].

In a previous study we found that Drosophila melanogaster and other invertebrates (i.e., sea urchin, Caenorhabditis elegans, bee, wasp, mosquito and flour beetle) have only one mitoferrin gene, which is most likely a functional homolog of vertebrate mitoferrin 2 as invertebrates lack erythropoiesis [13]. Study of Drosophila mitoferrin (dmfrn) in insect cell culture showed that its dysregulation affects cellular iron homeostasis through the iron-sulfur cluster synthesis pathway [13].

Mitochondria play an important role during spermatogenesis. For example, defects in caspase activation involving the spermatogenesis-specific cytochrome gene $c y t$-c$d$ [14] or defects in mitochondrial fusion processes, involving the fuzzy onions gene product [15], result in male sterility with defects during spermatogenesis. Drosophila melanogaster testes are $2 \mathrm{~mm}$ long terminally blind tubes. Spermatogenesis starts at their apical tip where stem-cell divisions give rise to germ cells [16]. Each germ cell is contained in a cyst of two somatic cyst cells [17] and undergoes four mitotic divisions, resulting in 64 syncytical spermatids after meiosis [18]. Mitochondrial fusion processes in spermatids result in two giant mitochondrial derivatives per spermatid that furl up to form the nebenkern. During elongation, the mitochondrial derivatives unfurl along the flagellar axoneme. As the individualization complex progresses along the length of the spermatids, each spermatid is wrapped in its own membrane, the minor mitochondrial derivative is depleted of most of its material, and other organelles and most of the cytoplasm are removed from spermatids and accumulate in the cystic bulge, which is cast off at the end of the spermatids as a waste bag [18]. After coiling, individualized spermatids are released from their cyst and stored as mature sperm in the seminal vesicle.
Here we report on a function of $d m f r n$ during spermatogenesis and characterize its expression in different fly tissues as well as within testis. We show for the first time that the mitochondrial iron metabolism is essential during spermatogenesis.

\section{Results}

P-element insertion P\{lacW\}dmfrn ${ }^{S H 115}$ in the 5'untranslated region of $d m f r n$ results in male sterility

Previously we have identified $d m f r n$ (CG4963), the only Drosophila homolog of yeast Mrs3/4 and vertebrate mitoferrin2, and studied its role in cellular iron homeostasis in cell culture [13]. Little is known about the role of mitoferrin 2 in the whole organism.

To study $d m f r n$, we obtained four publicly available mutant alleles. Three are due to P-element insertions in the $5^{\prime}$ untranslated region ( 5 ' UTR) of the $d m f r n$ gene and one is a deficiency where $d m f r n$ and a small part of the region in its proximity are deleted $(D f(3 R) E D 6277$; Figure 1A).

The insertion site of $P\{l a c W\} l(3) S H 115^{S H 115}$ is downstream of the putative transcriptional start (about 252 bp;) but upstream of the start of the dmfrn-coding sequence (Figure 1A). It was recovered during a screen for recessive lethal genes [19], which would be in agreement with iron-sulfur clusters being essential co-factors [3] and the proposed general function of mitoferrin 2 in mitochondrial iron transport in non-erythroid tissues [11]. P-elements $P\{G T 1\} m f r n^{B 00456}$ and P\{Epgy2\}mfrn ${ }^{E Y 01302}$, are both located closer to the putative transcriptional start of $d m f r n$ (about 20 and 40 bp downstream, respectively, Figure 1A) and no phenotypes caused by the insertions themselves have been reported. $P\{$ Epgy 2$\} m f r n n^{E Y 01302}$ contains an UAS sequence upstream of $d m f r n$ and has been used in a gain of function screen, where overexpression of $d m f r n$ in developing muscle apodemes caused muscle misdevelopment [20].

While cleaning the fly lines from background mutations by outcrossing them with wild type flies (strain $\left.w^{1118}\right)$, lethality of $S H 115^{l(3) S H 115}$ flies was lost after only three generations. Therefore, it was unlikely that the reported lethality was caused by allele $S H 115^{l(3) S H 115}$ but by another unmarked mutation instead. Consequently, the allele $S H 115^{l(3) S H 115}$ should be referred to as dmfrnsH115.

To our surprise we failed to establish a homozygous stock of $d m f r n n^{S H 115}$ flies, as male flies were recessive sterile and sterility was completely penetrant. Female $d m$ frn $n^{S H 115}$ flies were fertile ( $100 \%$ fertile; $\left.\mathrm{n}=20\right)$, while male recessive sterility persisted even after outcrossing $d m$ frn $^{\mathrm{SH} 115}$ flies for 13 generations to $w^{1118}$ flies. To confirm that the allele $d m f r n^{S H 115}$ was indeed responsible for male sterility, a P-element excision screen, in which the P- 


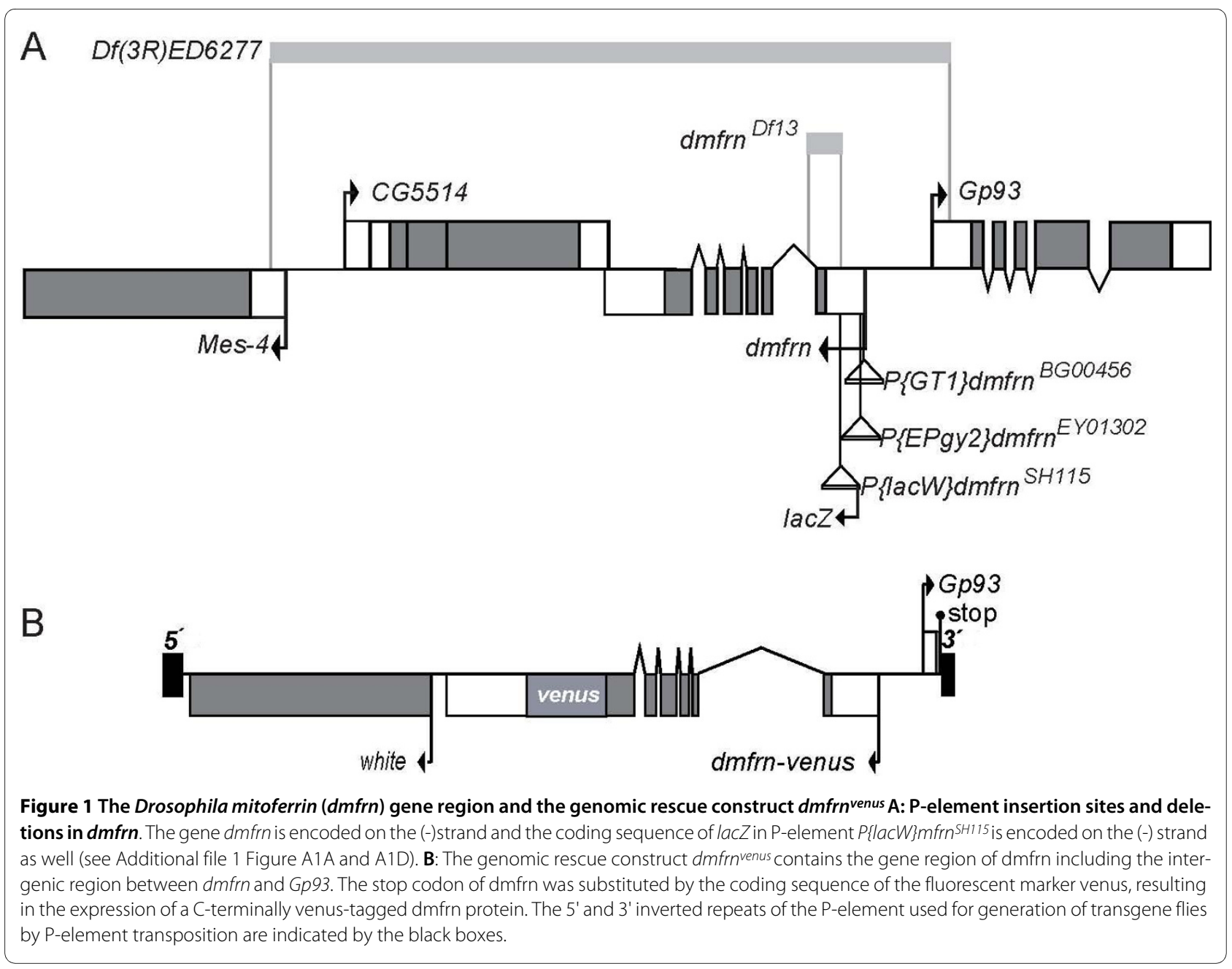

element was re-mobilized, was carried out [21]. Independent lines of flies that had lost the marker for the P-element were established and analyzed by PCR for precise and imprecise excisions (i.e., P-element leftovers or genomic deletions) (Table 1). All precise excisions in trans to $d m f r n$ SH115 rescued the recessive male sterility phenotype (Table 1). These results show that $P\{l a c W\} d m f^{\prime} n^{S H 115}$ causes male sterility.

We also analyzed fertility in male flies that carried $d m f r n^{S H 115}$ in trans to $D f(3 R) E D 6277$ or the small deletion $d m f r n^{D f 13}$ (Figure 1A), which was recovered during the hop-out assay, as well as flies that carried $d m f r n^{D f 13}$ in trans to $D f(3 R) E D 6277$ or were homozygous for $D f(3 R) E D 6277$. All of these combinations of dmfrn mutations resulted in male sterility (Table 2 ). To further support the role of $d m f r n$ in male fertility and to rule out that $P\{l a c W\} d m f r n$ SH115 interferred with enhancer or repressor elements of other nearby genes, and thereby resulted in male sterility, we performed a genomic rescue experiment using a transgene fly line, which contains the gene region of $d m f r n$ into which we had inserted a C-terminal venus tag (Figure 1B). This analysis showed that male ste-

Table 1: Transposon excision: test of fertility of white eyed flies (n.a. not analyzed).

\begin{tabular}{|c|c|c|c|}
\hline Hop-out class & Total number of lines & $\begin{array}{l}\text { Homozygous } \\
\text { fertile }\end{array}$ & $\begin{array}{l}\text { Hop-out/SH115 } \\
\text { fertile }\end{array}$ \\
\hline precise excision & 11 & 10 & 11 \\
\hline transposon leftovers & 7 & 1 & 1 \\
\hline deletion & 3 & n.a.* & n.a. \\
\hline
\end{tabular}

*the lines exhibited different degrees of lethality 
Table 2: dmfrnvenusB32 rescues male sterility of homozygous dmfrn ${ }^{\mathrm{SH} 115}$ as well as transheterozygote $d m f r n{ }^{S H 115}$ / dmfrn'f13, dmfrn SH115/Df(R3)ED6277 and dmfrnDf13/ Df(R3)ED6277 male flies but not homozygous Df(R3)ED6277 male flies.

\begin{tabular}{|c|c|}
\hline Genotype & $\%$ fertile flies \\
\hline \multicolumn{2}{|l|}{ without rescue construct } \\
\hline$S M 1 C y /+; d m f r n n^{S H 115}$ & $0(n=37)$ \\
\hline SM1 Cy/+;dmfrn SH115/dmfrn Df13 & $0(n=50)$ \\
\hline SM1 Cy/+;dmfrn SH115/Df(3R)ED6277 & $0(n=30)$ \\
\hline SM1 Cy/+; dmfrn Df13/Df(3R)ED6277 & $0(n=10)$ \\
\hline SM1 Cy/+;Df(3R)ED6277 & $0(n=17)$ \\
\hline \multicolumn{2}{|l|}{ with rescue construct } \\
\hline$d m f r n n^{\text {venusB32/+; } d m f r n} n^{S H 115}$ & $97(n=33)$ \\
\hline$d m f r n^{\text {venusB32/+; }} d m f r n^{S H 115} / d m f r n n^{D f 13}$ & $100(n=25)$ \\
\hline$d m f r n^{\text {venusB32/+; } d m f r n}{ }^{S H 115 / D f(3 R) E D 6277}$ & $97(n=30)$ \\
\hline$d m f r n^{v e n u s B 32 /+; d m f r n}$ Df13/Df(3R)ED6277 & $100(n=37)$ \\
\hline$d m f r n$ venusB32/+; Df(3R)ED6277 & $0(n=22)$ \\
\hline
\end{tabular}

rility of homozygous $d m f r n^{S H 115}$ flies as well as sterility of $d m f r n^{S H 115 / d m f r n}{ }^{D f 13 f l i e s,} \quad d m f r n^{S H 115 / D f(3 R) E D 6277}$ flies, and $d m f r n n^{D f 13} / D f(3 R) E D 6277$ flies was rescued by the transgene $d m f r n^{\text {venus } 332}$ construct (Table 2). However, male sterility of homozygous $D f(3 R) E D 6277$ males was not rescued by $d m f^{2} n^{\text {venusB32 }}$ (Table 2), indicating that the sterility of homozygous $D f(3 R) E D 6277$ males is likely caused by the deletion of one of the three other genes affected by the larger deletion. According to the FlyAtlas [22], the uncharacterized gene CG5514 is expressed in nerve tissue, ovaries and testes and is therefore the best candidate for male sterility of dmfrnvenusB32/+; $D f(3 R) E D 6277$ flies. Inspection of testis squashes in these flies showed larger Nebenkerns associated with several normal sized nuclei (see Additional file 1 Figure A2), which is a sign for cytokinesis defects [23]. This indicates that CG5514 might be involved in cytokinesis during spermatogenesis.

The fact that the emergence of homozygous Df(3R)ED6277 adult flies was lower than expected (6\% instead of $\sim 33 \%$; Figure 2A), indicates that Df(3R)ED6277 causes lethality in addition to sterility. Flies with any of the three P-element insertions in trans to Df(3R)ED6277 did not show any obvious signs of lethality (Figure 2B). Male flies with the genomic rescue construct dmfrnvenusB32 on the second chromosome, and either the small or the large deletion on the third chromosome, were crossed to female Df(3R)ED6277/TM6c flies, and offspring were scored. The fractions of dmfrnDf13/ Df(3R)ED6277 and homozygous Df(3R)ED6277 flies that carried the rescue construct ( $27 \%$ and $18 \%$, respectively) were larger than the fractions of flies without the rescue construct (13\% and $12 \%$, respectively) (Figure 2A). These results show that the deletion of dmfrn results in partial lethality, indicating an essential role of dmfrn during development to adulthood.

As spermatogenesis uses many processes that are also needed during the normal development of an organism (i.e., cell proliferation, growth and morphogenesis) and the male sterility phenotype of $d m f r n^{S H 115}$ flies was completely penetrant, we continued investigating the role of dmfrn during spermatogenesis.

\section{dmfrn $\mathrm{SH}^{115}$ causes elongation defects during spermatogenesis}

Male sterility can result from defects in mating behavior, abnormal anatomy of the sexual organs or defects during spermatogenesis [24]. Since homozygous dmfrn ${ }^{S H 115}$ males were observed mating, behavioral abnormalities were ruled out as the cause of male sterility. Dissection of male flies revealed that the testes of homozygous $d m f r n n^{S H 15}$ flies exhibited a defect during spermatogenesis of variable intensity resulting in the absence of mature sperm, whereas heterozygous testes looked normal and contained motile mature sperm (Figure 3A arrow head). The severity of the spermatogenesis defect in homozygous $d m f r n^{S H 115}$ flies ranged from testes that lacked properly elongated spermatids to those that looked almost like wild type (WT) testes but lacked mature sperm (Figure 3B to 3D shows some examples).

By analyzing testes squashes we found that $d m f r n S H 115$ spermatocytes (Figure 3F) and onion stage spermatids (Figure 3H) looked normal, whereas later stages of spermatids showed different defects. We found signs for delayed spermatid elongation (Figure 3J and 3K). Often, we observed abundant white structures of unknown origin in spermatid bundles (Figure 3L and 3L'). Sometimes mitochondrial derivatives of elongating and elongated spermatids had bulby protrusions (Figure $3 \mathrm{~N}$ and $3 \mathrm{~N}^{\prime}$ arrow head) that are reminiscent of unelongated or improperly elongated mitochondrial derivatives. We also observed elongated spermatids that appeared normal, but never mature sperm. This is supported by the finding that all $d m f r n^{S H 115}$ males were completely sterile. All of these results are indicative of an elongation defect in $d m$ frn $^{\mathrm{SH} 115}$ testes.

At the ultrastructural level, elongated spermatids of $d m f_{r n} n^{S H 15}$ testes exhibited great morphological defects compared to those of WT testes. Spermatid cysts were unorganized (compare Figure $4 \mathrm{~A}$ and $4 \mathrm{C}$ to Figure $4 \mathrm{D}$ and $4 \mathrm{I})$. In testes of $d m f r n^{S H 115}$ flies, the major and minor mitochondrial derivatives, associated with axonemes 


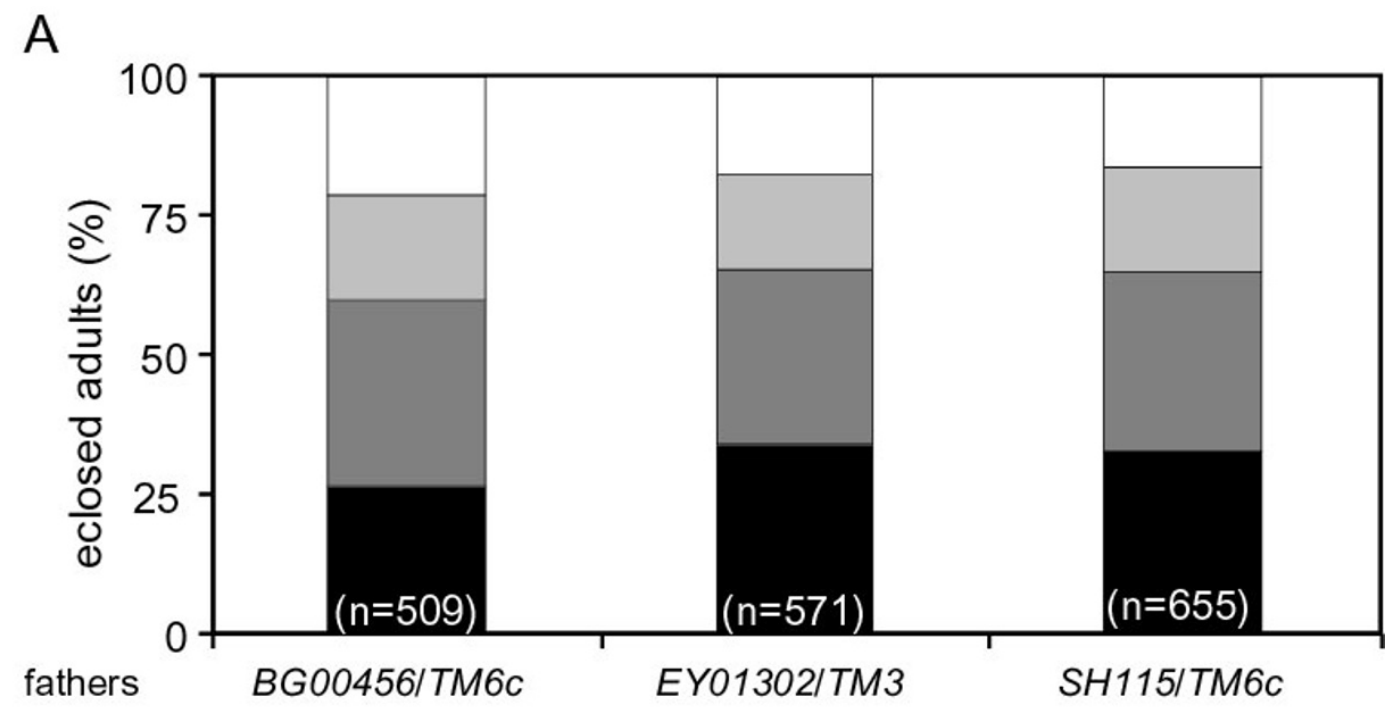

offspring $\square$ male balanced $\square$ female balanced $\square$ male $\square$ female

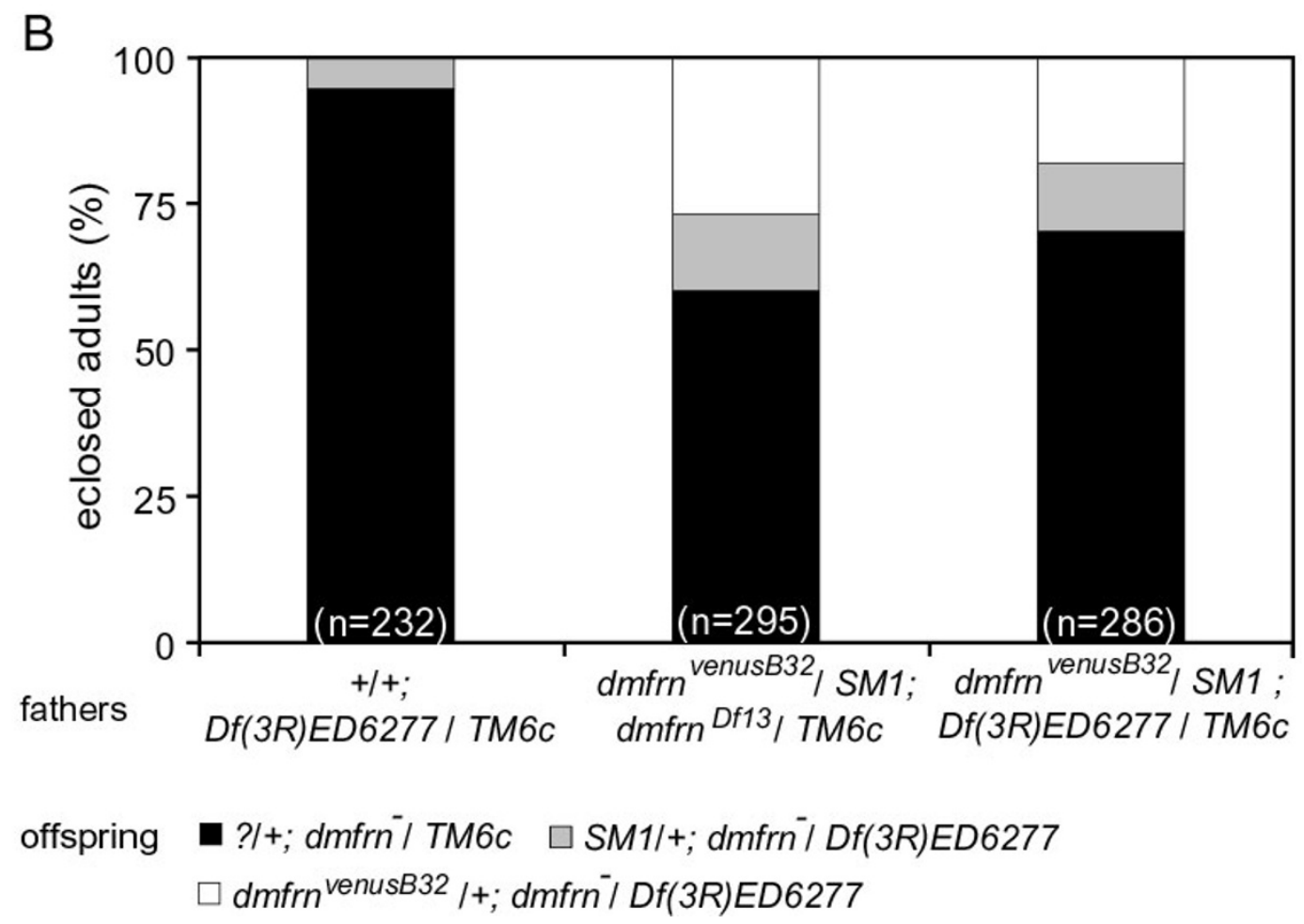

Figure 2 Development of $\boldsymbol{d m f r n}$ mutants to adulthood. Female Df(3R)ED6277/TM6c flies were crossed to male flies of the indicated genotype and allowed to lay eggs for three days. Numbers in parentheses show the total number of flies assayed per genotype. (A) Eclosed adult flies were collected and genotyped according to their genotypic markers. (B) Eclosed flies were sexed and genotyped according to their genotypic markers during an eclosion period of seven days. Male and female balanced: flies heterozygous for dmfrn mutations. Male and female $d m f r n:$ the indicated $d m f r n$ mutation in trans to $D f(3 R) E D 6277$. 

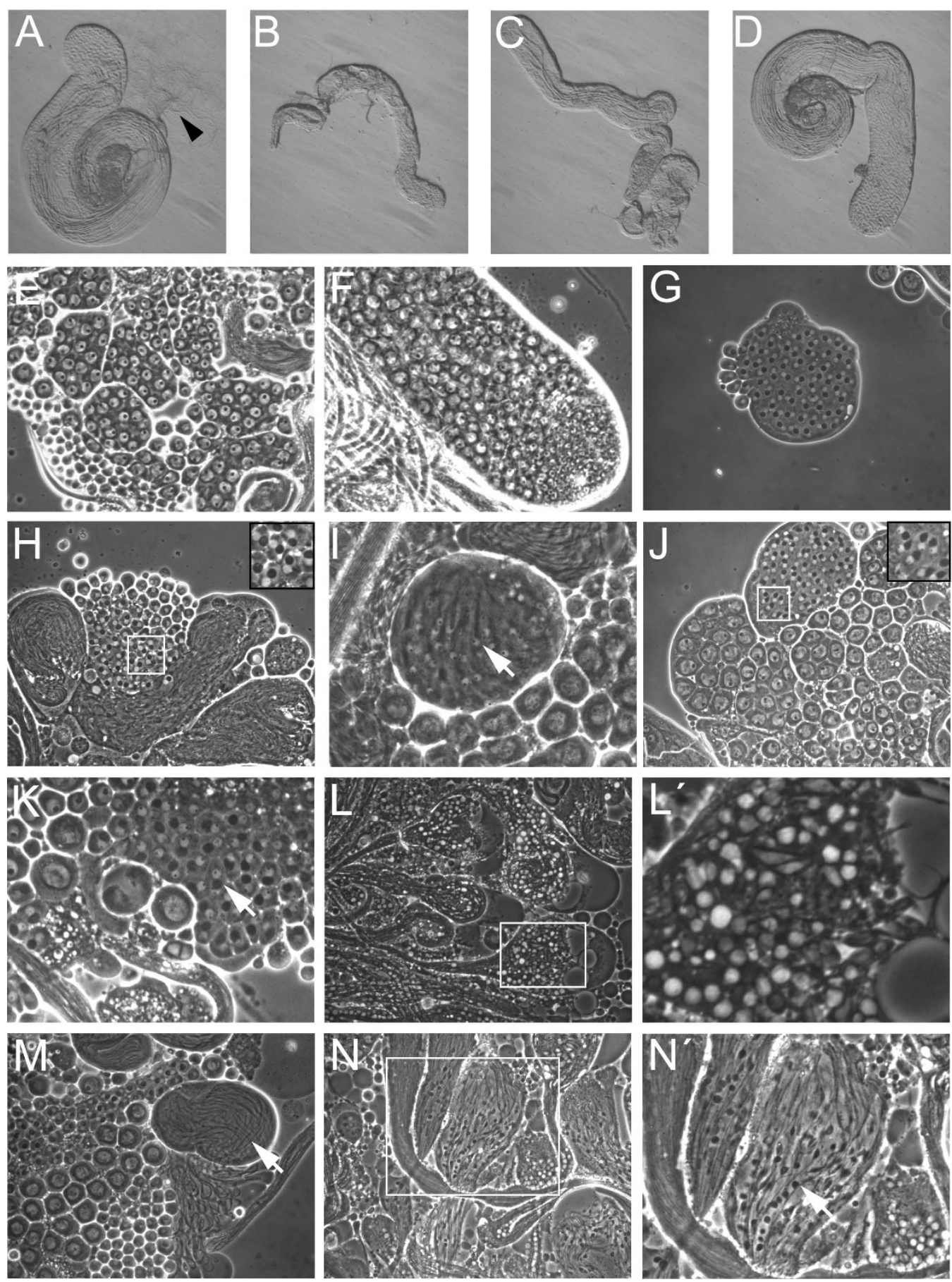

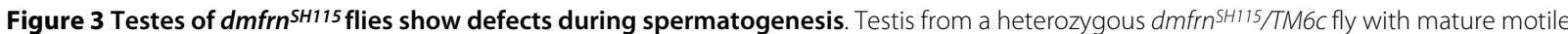
sperm (A arrow head). Testes from homozygous $\mathrm{dmfrn}^{5 H 115}$ flies lack mature sperm, can be smaller (B) than WT, often have fewer elongated spermatids

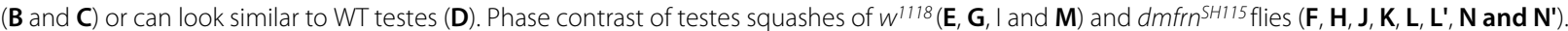
Spermatocytes $(\mathbf{F})$ and onion stage spermatids $(\mathbf{H})$ of dmfrn ${ }^{S H 115}$ tesis did not show any obvious defects and are indistinguishable from WT spermatocytes (E) and onion stage spermatids (G). Early elongating spermatids of $d m f r n n^{5 H 115}$ testis show signs of delayed elongation ( $\mathbf{J}$ and $\mathbf{K}$; the arrow indicates the dark spot on the nucleous, which is characteristic for elongating spermatids, as can be seen in WT (I)). Spermatids of $d m f r n n^{5 H 115}$ testes frequently contained white spherical objects ( $\mathbf{L}$ and $\mathbf{L}^{\prime}$ ). Membrane blebbing was observed on mitochondrial derivatives of elongating spermatids of dmfrn ${ }^{5 H 115}$ testes ( $\mathbf{N}$ and $\mathbf{N}^{\prime}$ arrow) but not on WT testes (M, arrow) 

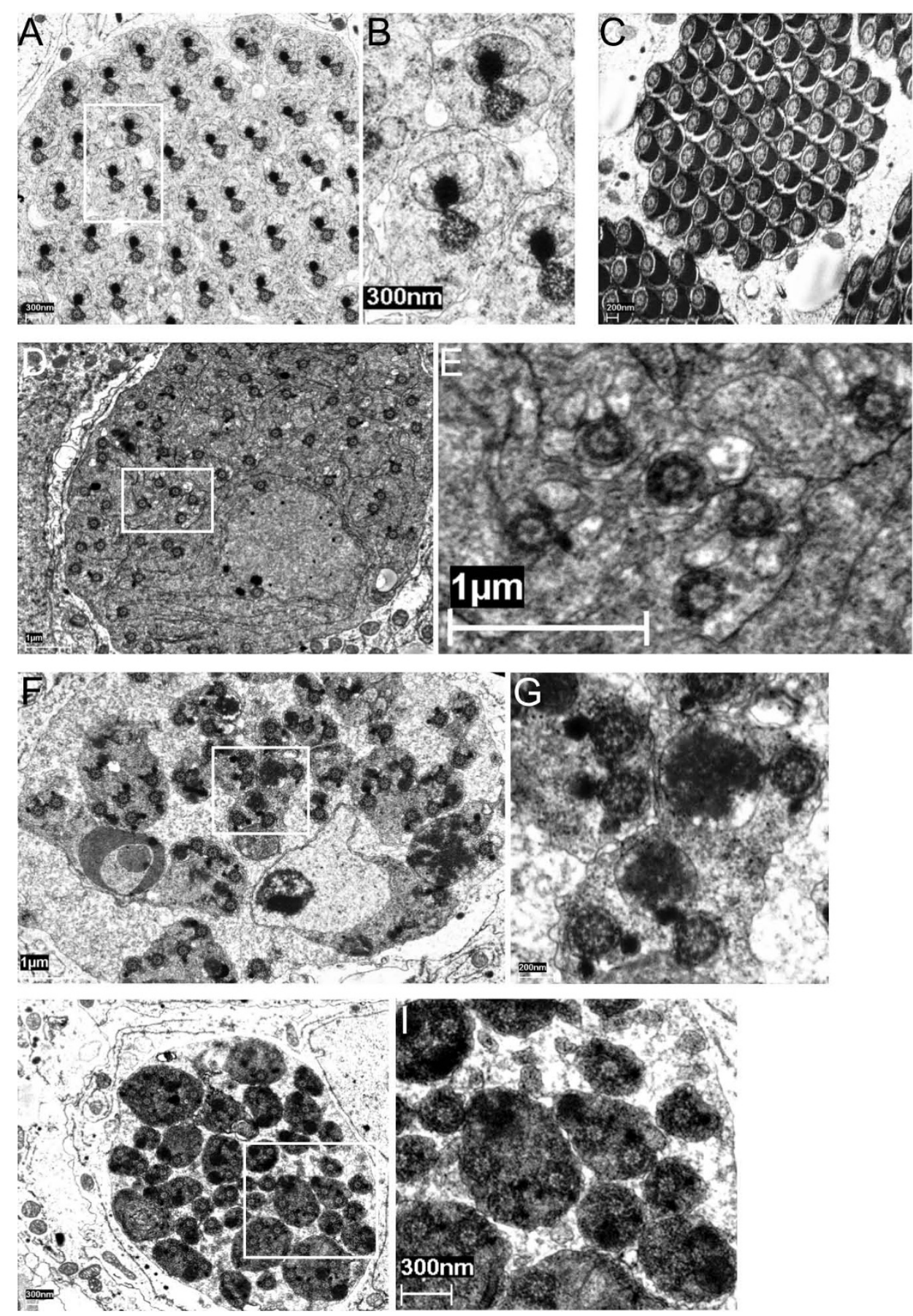

Figure 4 Spermatids from $\mathbf{d m f r n} \mathrm{SH}^{115}$ flies show defects in the maturation of the mitochondrial derivatives. Transmission electron micro-

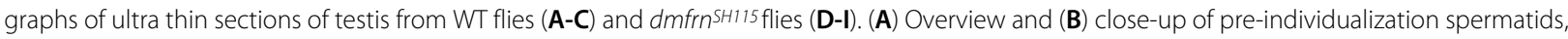
showing the symmetric distribution of the spermatids within the cyst. The major mitochondrial derivative can easily be distinguished from the minor mitochondrial derivative by the accumulation of the electron dense stain. (C) Overview of individualized spermatids, which shows parachrystalline symmetry, a clearly visible major mitochondrial derivative and depletion of cytoplasm. (D) Overview and (E) close-up of $d m^{\prime}$ rn $^{\text {SH115 }}$ pre-individualiza- $^{-}$ tion spermatids. No symmetry of the spermatids can be observed and accumulation of the paracrystalline structure to only a few major mitochondrial derivatives can be seen. $(\mathbf{F}$ and $\mathbf{H})$ Overview and $(\mathbf{G}$ and $\mathbf{I})$ close up of spermatids from $\mathrm{dmfrn}^{\mathrm{SH}}{ }^{115} \mathrm{flies}$. Several axonemes are within the same spermatids and the accumulation of the paracrystalline structure within mitochondria is very heterogeneous. 
were impossible to distinguish from one another in many cases. Often, one axoneme was associated with two mitochondrial derivatives of similar size, and both had accumulated the paracrystalline structure (Figure 4G), which is normally typical for the major mitochondrial derivative $[25,18]$. In some cases, one such mitochondrial derivative was extremely enlarged and contained heterogeneous accumulations of the paracrystalline structure (Figure $4 G$ ). Furthermore, in the same cyst the number of spermatids wrapped in the same membrane was not constant (Figure $4 \mathrm{H}$ ), indicating that if individualization occurred, it was defective.

Defects during spermatogenesis can either be primary defects, which are a direct consequence of a mutation or they can be secondary defects, which are the result of primary defects [23]. To find out whether possible defects during individualization, as indicated by the above TEM results, were preceded by earlier defects that are independent of the elongation defects that we observed under phase contrast, we analyzed whole mount testis of $d m f_{r n}{ }^{S H 115}$ flies by confocal laser scanning microscopy. Nuclei and f-actin were stained with DAPI and rhodamin phalloidin, respectively. In WT testes, nuclei of elongated spermatids were needle-shaped and located at the base of the testis in parallely packed bundles (Figure 5A, DAPI) and individualization complexes present at different positions of elongated spermatids were apparent from well organized actin-cones (Figure 5A, RhoPha). Even though nuclei of spermatids from $d m f r n^{S H 115}$ testes were needleshaped, they were often scattered over large areas of the length of the testes (Figure 5B, DAPI) or formed fuzzy bundles (Figure 5C, DAPI). Despite the presence of parallel organized nuclei in some mutant spermatid bundles, we did not observe actin-cones in the mutant testes we analyzed (Figure 5B and 5C, DAPI). However, in one testis we observed the associaton of f-actin with nuclei, which might be indicative for the formation of an individualization complex (Figure 5C). So, even though we could not see any actin cones, it may not be ruled out that some spermatids did form individualization complexes and underwent partial individualization. All defects observed in $d m f n^{S H 115}$ testes were rescued in testes of

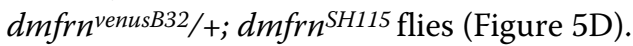

A parallel organization of the nuclei at the start of individualization is required for the successful assembly of functional individualization complexes. From the above results, it is therefore likely that the defects observed by TEM in $d m f r n^{S H 115}$ testes are the result of the elongation defects.

\section{dmfrn is ubiquitously expressed with slightly higher expression in testes}

Mitochondrial ferritin, an iron storage protein, is most abundantly expressed in testes of mice [26,27] and flies
[28], indicating that the mitochondrial iron metabolism, in particular, might play a role during spermatogenesis in insects and mammals.

According to the FlyAtlas [22], dmfrn expression is lowest in testes, which was rather unexpected in light of our findings. This prompted us to verify $d m f r n$ expression in different tissues by RT-RT PCR. We isolated RNA from heads, thoraxes, guts, malpighian tubules and testes of 23 days old virgin male flies and analyzed the expression levels of $d m f r n$ and other iron metabolism related genes (i.e., frataxin homolog (fh), Fer $1 \mathrm{HCH}$, Fer $2 \mathrm{LCH}$ and Fer3HCH) as well as the house keeping gene RP49.

Mitochondrial ferritin $(\mathrm{Fer} 3 \mathrm{HCH})$ was expressed at extremely low levels in all tissues except for testes where its message was about eight times more abundant than in whole fly homogenates (Figure 6). This expression pattern is in agreement with that of a previous report [28] and is similar to the FlyAtlas expression data (about 10 times more transcript in testes, compared to whole flies of both sexes).

Transcripts of Fer $1 \mathrm{HCH}$ and $\mathrm{Fer} 2 \mathrm{LCH}$ were enriched about twofold in the gut and malpighian tubules but not in testes (Figure 6), which again is similar to the pattern reported in the FlyAtlas. Ferritins of Drosophila are involved in iron storage and iron transport (for a review on insect iron metabolism see [29]), and accumulate in the iron region of the midgut of iron loaded flies [30]. Higher expression of ferritins in the gut could therefore either be an indication for the mobilization of food iron to other tissues or iron storage.

The expression patterns of $d m f r n$ and $f h$ in our analysis (Figure 6) diverged from the FlyAtlas data. In all tissues, except for testes, both were expressed at levels similar to that of whole flies. In testes, message abundance of $d m f r n$ and $f h$ were about two-fold higher (Figure 6).

\section{dmfrn is expressed at increased levels in spermatids}

The above mentioned results indicate that $d m f r n$ expression in testes is higher than in other tissues. Testes contain both somatic cells; i.e, cyst cells and cells of the testis sheath, as well as germline cells of different developmental stages. Mitochondrial aggregation and formation of the giant mitochondrial derivatives take place in spermatids [24] and since we observed spermatid elongation defects, we suspected that mitoferrin expression could occur before or during this stage.

$P\{l a c W\} d m f r n$ SH115 contains the coding sequence (CDS) of the $\beta$-galactosidase gene (lacZ) [31] on the same strand as dmfrn (Figure 1A and see Additional file 1 Figure A1) allowing the visualization of the expression pattern of $d m f r n$ in testes through X-gal staining. Blue stain was not detectable in testis of $w^{1118}$ flies, which were used as a negative control (Figure 7A left). In testis from heterozygous $d m f r n^{S H 115}$ flies, blue stain accumulated in 

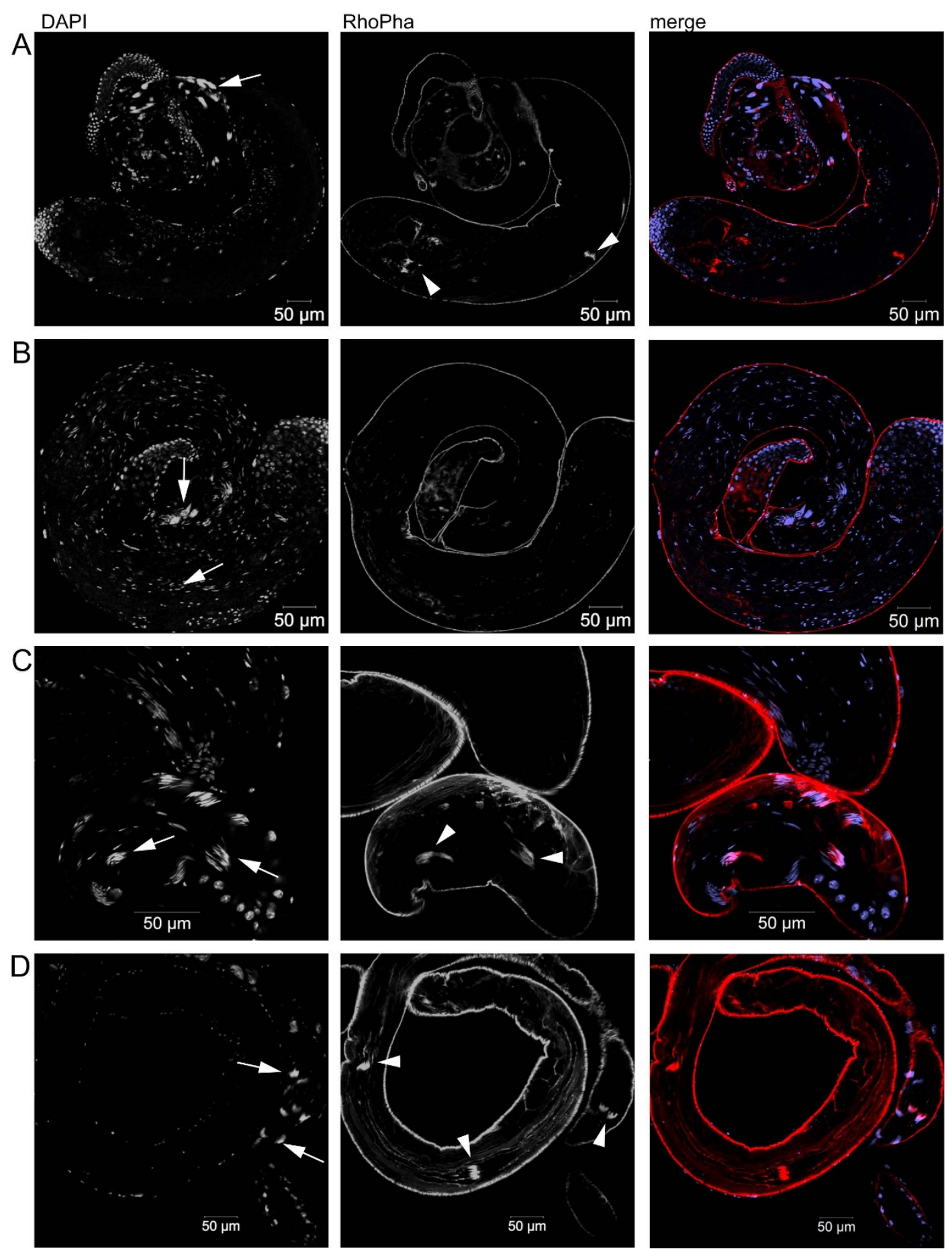

Figure 5 Spermatid elongation defects in testis of $\mathbf{d m f r n}^{\text {SH115 }}$ flies. Nuclei were stained with DAPI (blue) and actin filaments were stained with rhodamine phalloidin (red). (A) Nuclei of elongated spermatids in WT testis are needle shaped and arranged in parallel (arrow). Individualization com-

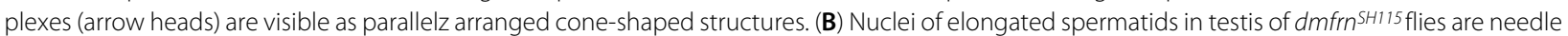
shaped but scattered (arrows). Individualization complexes were not observed. (C) Higher magnification of the base of a testis from dmfrnSH115 flies, showing the scattered, but needle shaped nuclei of elongated spermatids. Two nuclei bundles are associated with f-actin structures (arrows), which might be newly forming individualization complexes. (D) Parallel, needle shaped nuclei (arrows) and individualization complexes (arrow heads) are

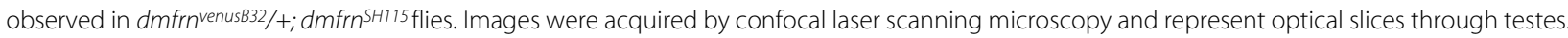




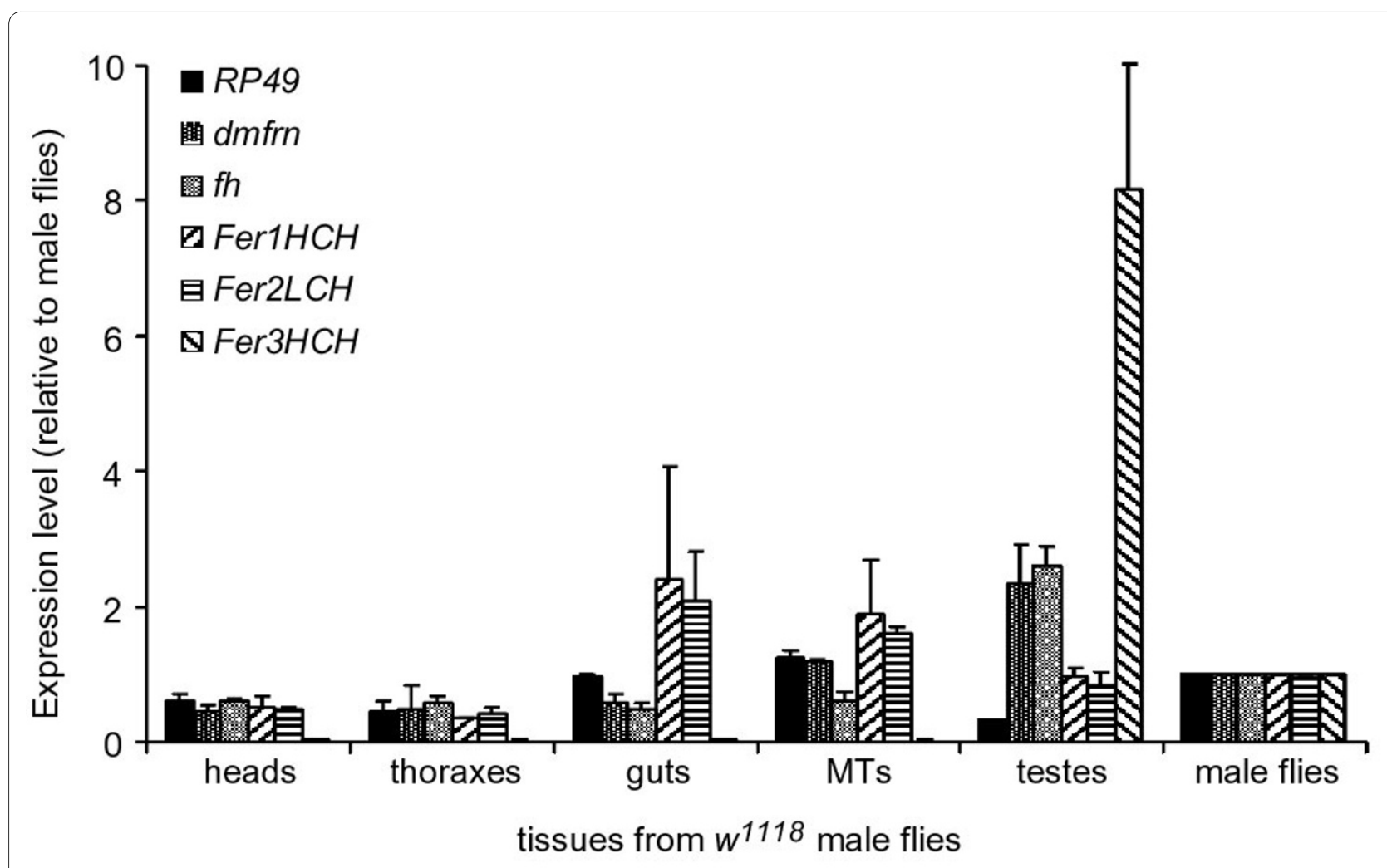

Figure 6 Expression pattern of iron metabolism related genes in tissues of adult males. CDNA was prepared from RNA extracted from dissected tissues of 2-3 days old adult male $w^{1118}$ flies. MT $=$ malpighian tubules. Gene expression in the tissues is relative to that of gene expression in whole flies. The data represent the mean of two independent experiments.

spermatids and was absent from spermatocytes and the rest of the genitalia (Figure 7A middle). X-gal staining of small testis from homozygous $d m f r n^{S H 115}$ flies showed the same pattern and only a few spermatids were elongated to a small degree (Figure 7A right).

To verify the expression pattern of $d m f r n$ and to be able to study the localization of dmfrn protein during spermatogenesis in further detail, we examined testes from $d m f r n^{\text {venusB32 }}$ flies under the fluorescence microscope. First, we made sure that the signal of dmfrn-venus protein was clearly discernible from autofluorescence of testes by using $w^{1118}$ flies as a negative control (Figure 7B left). A clear signal of dmfrn-venus protein was visible in elongated spermatids (Figure $7 \mathrm{~B}$ right). This expression pattern is similar to the expression pattern of $d m f r n$ obtained through the expression of lacZ, confirming that $d m f r n$ is expressed late during spermatogenesis. Using confocal microscopy with increased gain, we were also able to detect dmfrn-venus protein in spermatocytes and the testis sheath (see Additional file 1 Figure 3).

At higher magnification, we observed that dmfrn-venus protein accumulated in elongated spermatids, the region of spermatid individualization, and that a large fraction was disposed of in waste bags (Figure 8A and 8B). Closer inspection of whole mount $d m f$ rn $^{\text {venusB32 }}$ testes, using confocal microscopy, confirmed these observations (Figure $8 \mathrm{C}$ to $8 \mathrm{E}$ ) and showed that dmfrn-venus protein abundance was increased in nebenkerns of onion stage spermatids and in elongated spermatids (Figure 8C). During spermatid individualization, dmfrn-venus accumulated in mitochondrial whorls in front of the actin cones of individualization complexes (Figure 8D). At the end of spermatid individualization, the bulk of dmfrn-venus had accumulated in cystic bulges and ended up in waste bags (Figure 8E).

\section{Male fertility of hypomorph dmfrn mutants depends on food iron levels}

Yeast MRS3/4 double deletions only cause a growth defect on low iron medium [10]. We were therefore interested to see whether food iron levels had any effect on fertility of the $m f r n^{S H 115}$ mutant. We also wanted to test if the fertility of flies with the two other P-element insertions, which could be kept as homozygous stocks on normal food, were influenced by iron availability.

To be able to quantify and compare fertility of the different P-element mutants, we wanted to use a genetic 


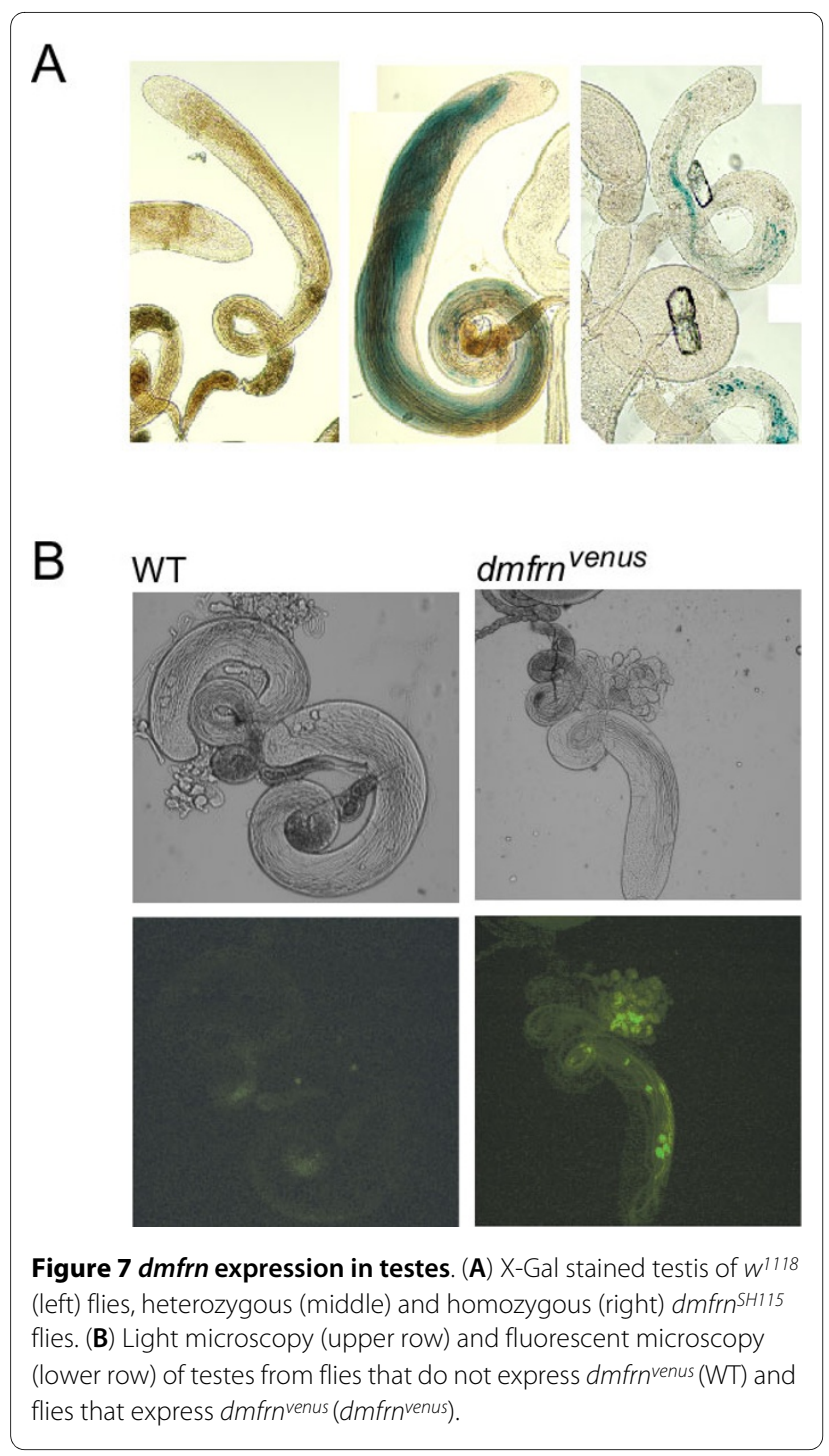

background that was as similar as possible. Therefore, we crossed male $d m f r n$ SH115/TM6c or dmfrn EY01302/TM3 or $d m r f n^{B G 00456 / T M 6 c}$ flies with female $D f(3 R) E D 6277 /$ $T M 6 c$ flies. Eggs were laid on low iron food (food containing the iron chelator BPS), normal food and high iron food $\left(\sim 2.5 \mathrm{mM} \mathrm{Fe}^{3+}\right.$ as ferric amonium citrate (FAC)) to allow development of offspring under these different iron conditions. Males that carried the respective mutant allele over $D f(3 R) E D 6277$ were collected and fertility was quantified.

$D f(3 R) E D 6277 / T M 6 c$ males raised on low iron food were completely fertile (Figure 9A), showing that iron starvation by itself did not cause male sterility.

Male sterility on normal food was low in dmfrn ${ }^{B G 00456 /}$ $D f(3 R) E D 6277$ flies, higher in $d m f n^{E Y 01302 /}$ $D f(3 R) E D 6277$ flies and complete in dmrfn ${ }^{S H 115 /}$ $D f(3 R) E D 6277$ flies (Figure 9A). Supplementation of food with iron increased fertility of dmfrn ${ }^{\mathrm{EYO} 1302 /}$
$D f(3 R) E D 6277$ flies strongly, while low iron conditions reduced fertility of $d m f r^{B G 00456 / D f(3 R) E D 6277}$ and $d m f r n{ }^{E Y 01302 / D f(3 R) E D 6277}$ males drastically (Figure 9A). $d m f r n^{S H 115} / D f(3 R) E D 6277$ male flies were completely sterile, regardless of the food they were raised on (Figure 9A).

We also observed testes of the different P-element mutants raised on low iron food. Testes of TM6c/ Df(3R)ED6277 flies, which were used as a control, looked like normal WT testes with abundant elongated spermatids and mature motile sperm in the seminal vesicle (Figure 9B), showing again, that low iron levels alone do not cause defects during spermatogenesis. Testes of $d m f r n^{B G 00456 / D f(3 R) E D 6277}$ and $d m f r n^{E Y 01302 /}$ $D f(3 R) E D 6277$ flies contained very few elongated spermatids (Figure 9C and 9D and Figure 9E and 9F, respectively), whereas $m f r n^{S H 115} / D f(3 R) E D 6277$ testes were very small and did not contain elongated spermatids (Figure 9G). This, in turn, shows again that $d m f r n^{S H 115}$, which is further downstream in the $5^{\prime}$ UTR of $d m f r n$, causes the strongest phenotype.

From these results, and the well established role of mitoferrins in the mitochondrial iron metabolism, we conclude that $d m f r n$ and the mitochondrial iron metabolism are essential for spermatogenesis.

\section{Discussion}

The yeast homologs of $d m f r n$ and frataxin homolog (fh) have been shown to genetically interact in yeast during iron-sulfur cluster (ISC) biosynthesis [9] and heme biosynthesis [8]. Previously, we have shown that $d m f r n$ rescues yeast MRS3/4 double mutants and that its overexpression alters cellular iron homeostasis [13]. In the current study we show that the mitochondrial iron metabolism plays a role during spermatogenesis for the first time directly, through the male sterility phenotypes caused by P-element insertions into dmfrn and the dependence of the hypomorph mutant on dietary iron, and indirectly, through the expression of $f h$ and $d m f r n$ in testes. This is even further supported by the high expression level of mitochondrial ferritin in testis (our results and [28]). The fact that frataxin, mitochondrial ferritin and $d m f r n / m i t o f e r r i n 2$ are all expressed at higher levels in testes of Drosophila and mammals [32,26,27,11,33], indicates that the involvement of the mitochondrial iron metabolism in spermatogenesis is very likely conserved from insects to mammals.

Early studies of mammalian spermatogenesis indicated a nutritional function of iron during spermatogenesis. In human seminal plasma, levels of transferrin, an ubiquitous iron transport protein in mammals, are correlated with sperm abundance [34]. Furthermore, transferrin is produced in Sertoli cells ("nurse cells") of mammalian testes and delivers iron to germinal cells [35-37]. Defects in 

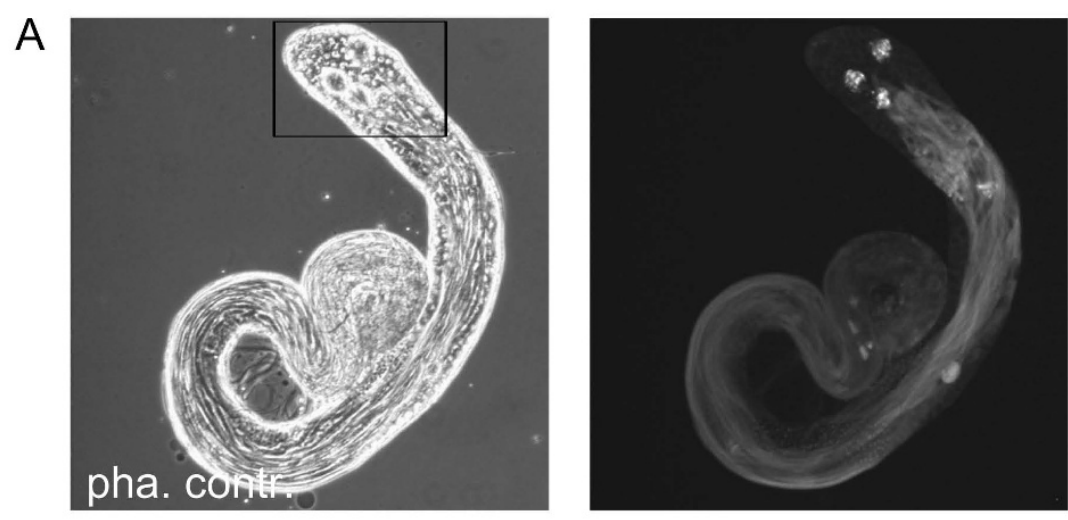

B
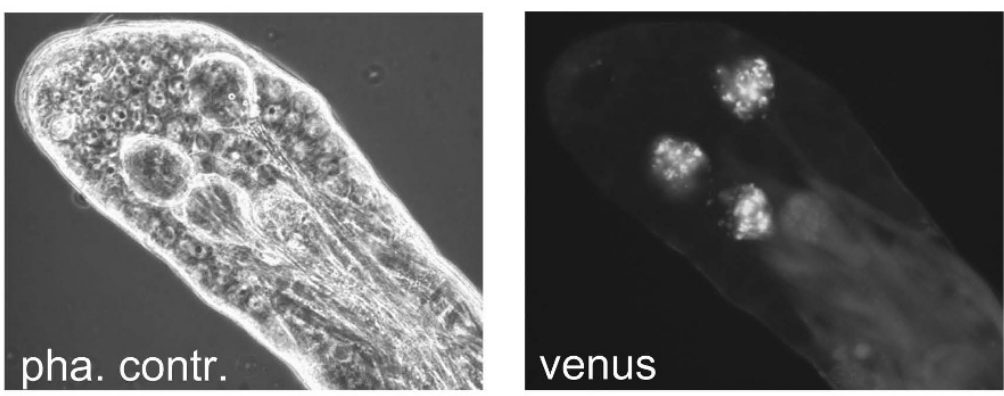

C
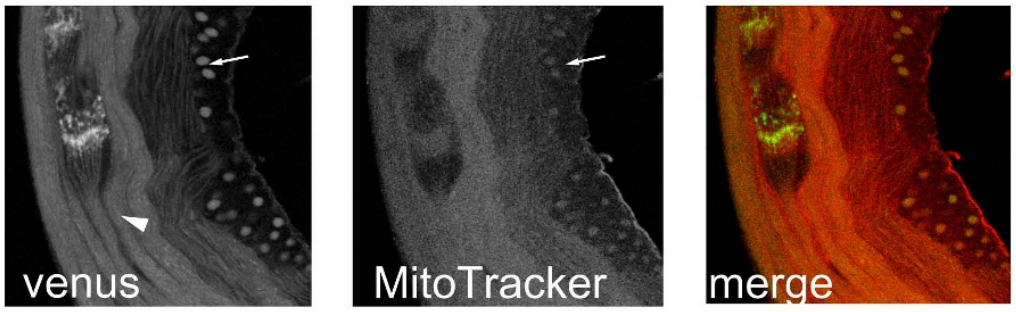

D
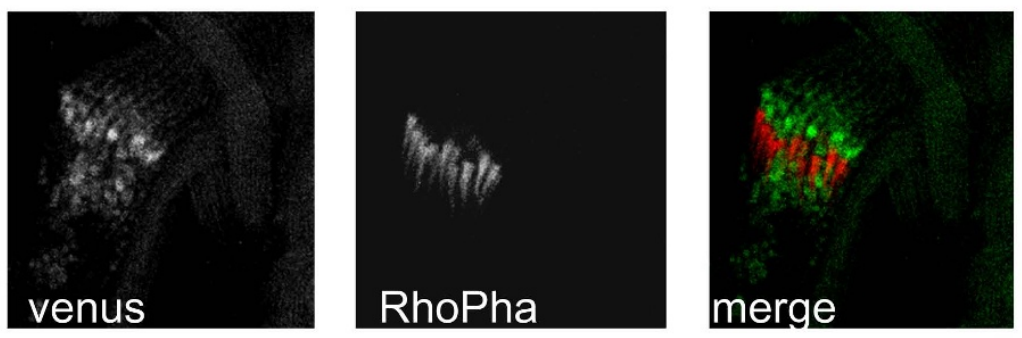

$E$
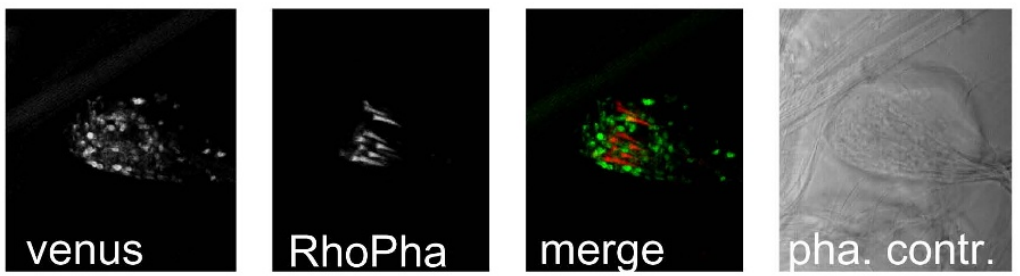

Figure 8 Localization of dmfrn-venus protein in testis using conventional microscopy ( $A$ and B) and confocal laser scanning microscopy (CE). (A) Phase contrast (pha. contr.) and fluorescence (venus, green) microscopy of a testis from a dmfrnvenusB32 fly. dmfrn-venus protein accumulates in elongated spermatids and waste bags. (B) Higher magnification of the area indicated in (A), showing the accumulation of dmfrn-venus in waste bags. (C-E) Confocal laser scanning microscope images of $d m f r n v e n u s B 32$ expression in spermatids. (C) Expression of dmfrnvenusB32 (venus, green) in whole mount testis. Mitochondria stained with MitoTracker Deep Red 633 (MitoTracker, red). Arrow: onion stage spermatids; arrow head: elongated spermatids. (D) Localization of dmfrn-venus inside the cystic bulge at the individualization complex. dmfrn-venus (venus, green) accumulates in mitochondrial whorls in front of the actin cones (RhoPha, red) of the individualization complex. (E) After individualization, dmfrn-venus (venus, green) accumulates in waste bags. 


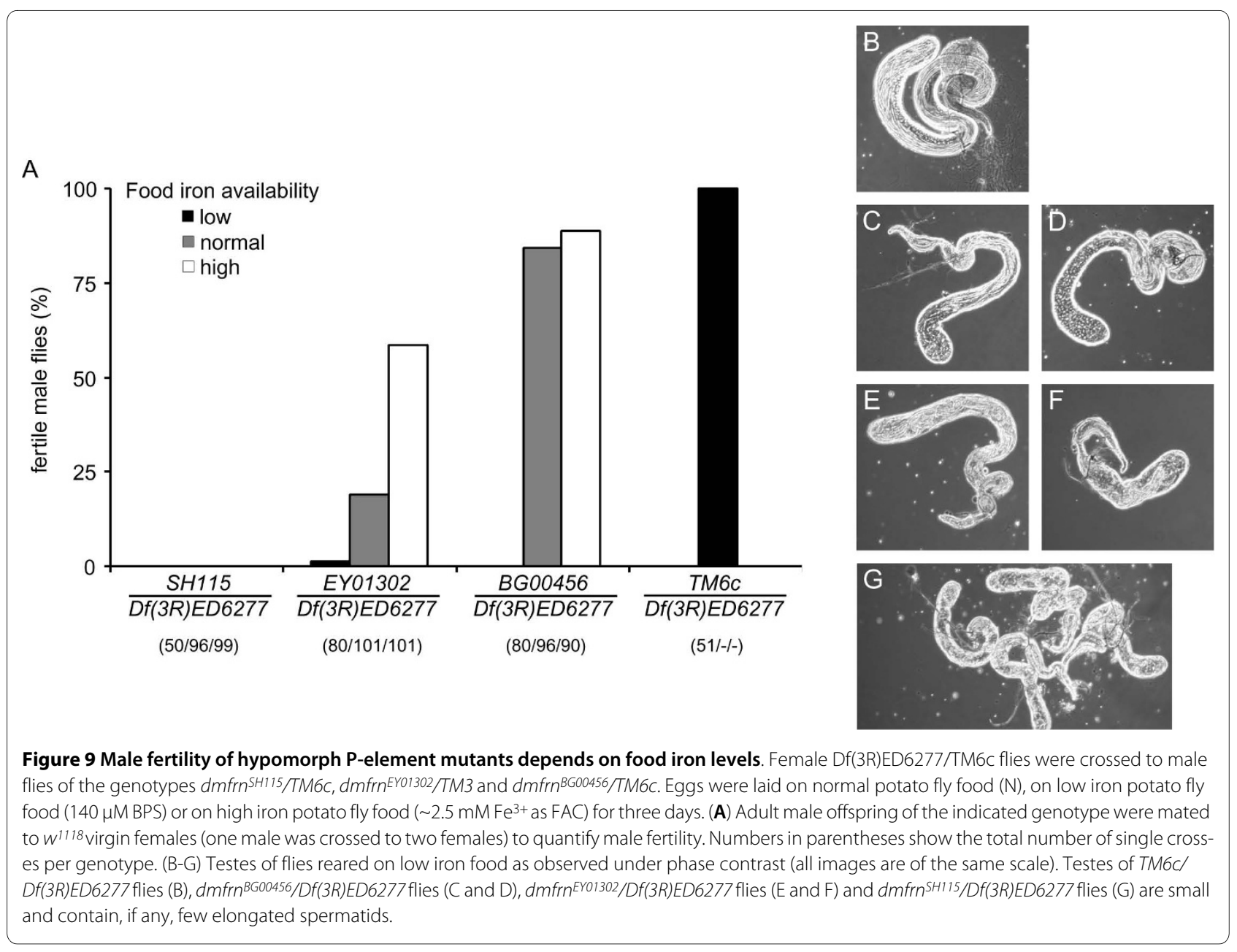

the mitochondrial iron metabolism, especially the ironsulfur cluster synthesis pathway, result in cellular iron accumulation [38-40]. Increased iron uptake and accumulation in yeast MRS3/4 mutants have been shown [41] and might occur in $d m f r n$ mutants. It has been shown that injection of iron into testes of rats results in sterility and tissue degeneration [42], and similar experiments with other metals do suggest that this might be a general effect of heavy metals [43]. However, the fact that nutritional iron loading of $d m f r n$ mutant flies rescued the weaker male sterility phenotypes and that iron starvation enhanced these phenotypes, indicates that the spermatogenesis defects in the testis of $d m f r n$ mutants is not the result of cellular iron overload. We interpret our results as further support for a nutritional function of iron during spermatogenesis. This would also be in agreement with the large variety within the testis development we observed (ranging from very small to WT-like testis that lack mature sperm).

The growth defect of yeast MRS3/4 double deletions develops only under iron limiting conditions, and it has been reasoned that other, still unidentified transporters with lower iron affinity could compensate for the lack in Mrs3/4p under iron replete conditions [10,6]. Deletion of $d m f r n$ results in partial lethality, whereas flies with the Pelement insertions in the 5 ' UTR of $d m f r n$ are viable. Therefore, we suggest that there is residual $d m f r n$ expression in the P-element insertion lines. In those lines with P-elements integrated closest to the putative transcriptional start site of $d m f r n$, expression might be high enough to allow nutritional iron loading to compensate for lower $d m f r n$ expression, while $d m f r n$ expression in $d m f r n$ SH115 testes would be insufficient to sustain spermatogenesis, even under iron loading conditions. Testes are heterogeneous microenvironments and the germ cells are contained within a pair of cyst cells during all stages of development, and the function of cyst cells in Drosophila is poorly understood. Therefore, it could very likely be that access of germ cells to iron is controlled by cyst cells, and that even iron loading of $d m f r n^{S H 115}$ flies cannot provide enough iron for a low affinity transporter to compensate for the lack of dmfrn in $d m f r n^{S H 115}$ testes.

Several properties and phenomena of spermatogenesis are likely to rely on mitochondrial iron metabolism: (i) 
Mitochondria of spermatids aggregate to form the giant nebenkerns through fusion processes that depend on mitochondrial activity [44], which in turn depends on the activity of the respiratory chain. Several complexes of the respiratory chain contain heme or ISC or both as prosthetic groups, linking energy metabolism directly with the mitochondrial iron metabolism. Insufficient $d m f r n$ expression could lead to defects in the energy metabolism of the giant mitochondria and interfere with mitochondrial dynamics. (ii) Spermatids undergo dramatic morphological changes as they elongate to a length of almost $2 \mathrm{~mm}$. This process is most likely very energy craving and, therefore, well functioning electron transport chains should be essential. If the energy metabolism is corrupted, elongation is likely to stop or slow down. (iii) Spermatids undergo an apoptosis-like processes during their individualization. The testis specific variant of the heme protein cytochrome c, encoded by the gene $c y t-c-d$, has been shown to be essential for spermatid individualization $[45,14]$ and its function might be sensitive to heme deficiency. Even though we observed individualization defects that could hint to defects in the apoptosislike process in spermatids of $d m f r n$ mutants, it cannot be ruled out that preceding defects during spermatid elongation are the underlying cause.

The exact functions of the major and minor mitochondrial derivatives of insect sperm are not clear and several different hypotheses exist. Mitochondrial derivatives may be extremely efficient mitochondria or are degenerated mitochondria or modulate the undulation of the sperm tail in a species specific manner [25]. During spermatid individualization, a large part of the minor mitochondrial derivative is removed from spermatid tails and is disposed of in waste bags [18] and we found that a large fraction of dmfrn follows this portion of mitochondria. As the sperm tail is stripped from all of its organelles, except for the remaining part of the mitochondrial derivatives, mitochondrial transport is very likely to become obsolete. On the other hand, remaining mitochondrial carriers could clean the cytoplasm from left-over substrates. In Drosophila, ferritin resides in the endoplasmatic reticulum (ER) and can be secreted [46]. Using testes of Fer $1 \mathrm{HCH}^{\mathrm{G} 188}$ flies that express GFP-tagged Ferritin Heavy Chain Homolog protein [30], we were able to identify ferritin in close proximity to mitochondrial whorls and its accumulation in waste bags (see Additional file 1 Figure A4). The close proximity of the ER to mitochondrial whorls could be an indication of iron transfer from the Fer1HCH/FerLCH pool to mitochondria to maintain a functional respiratory chain and active cytochrome-c-d.

A recently published article reports that the copper transporter Ctr1C in Drosophila, is essential for male fertility in a $C \operatorname{tr} 1 B$ mutant background [47]. Furthermore,
Ctr1C locates to the cytoplasmic membrane and is expressed in spermatids and elongating spermatids [47], indicating that metals, in general, play an important role during spermatogenesis.

\section{Conclusions}

From our findings we conclude, that Drosophila mitoferrin and the mitochondrial iron metabolism are essential during spermatogenesis. Drosophila and mammalian spermatogenesis have several processes in common [48] and genes involved in the mitochondrial iron metabolism are expressed in testis of both vertebrates and Drosophila. Therefore, it is not unlikely that our findings are applicable for vertebrates as well. Our study provides a first insight and tools in the form of characterized fly mutants, that will aid further investigations concerning the role of iron, and specifically mitochondrial iron metabolism during spermatogenesis.

\section{Methods}

Fly strains

$w^{1118}$ and $w^{1118} ; V n o / T M 6 c S b$, Tb flies were obtained from M.S. Dushay (Illinois Institute of Technology) and $w^{1118}$; wg/CyO; $\Delta 2-3 \mathrm{Sb} / \mathrm{TM} 6 c$, Tb flies and $w^{1118}$; Sco/ $S M 1, C y ; V n o / T M 3, S b$ flies were obtained from P. Kylsten (Södertörns Högskola, Sweden). l(3)SH115 and $D f(3 R) E D 6277$ flies were obtained from Szeged Drosophila stock center, Hungary. Stocks of $d m f r n{ }^{E Y 01302}$ and $m f^{B} n^{B G 00456}$ flies were obtained from the Bloomington Drosophila stock center, USA. $w^{1118}$; Sco/SM1, Cy; Vno/ $T M 6 c, S b T b$ were made by conventional fly genetics. We genotyped dmfrn $n^{S H 115}$, (SH115l(3)SH115) dmfrnEY01302 and $m f n^{B G 00456}$ flies to confirm the stocks (see Additional file 1 , additional methods for genomic DNA extraction and PCR protocol).

The $\sim 11 \mathrm{kbp}$ large deletion $D f(3 R) E D 6277$ at cytogenic map position $98 \mathrm{~B} 6$ was generated by recombination as part of the DrosDel project [49,50]. It removed genes $d m f r n$ and CG5514 completely and parts of the putative 5' UTRs of Mes-4 and Gp93. Because Df(3R)ED6277 was uncharacterized and unverified, we performed PCR confirmation (see Additional file 1, additional Figure A1B and $\mathrm{A} 1 \mathrm{~F}$ ) as proposed by Ryder et al. [50] and sequenced the products, confirming the integrity of the recombination product.

Flies were kept on standard potato sucrose medium in a $12 \mathrm{~h} / 12$-h light/dark cycle. Fly stocks were kept at $18^{\circ} \mathrm{C}$. Experiments and crosses were carried out at room temperature $\left(22-25^{\circ} \mathrm{C}\right)$.

For histostaining and other microscopic work, adult male flies were separated from female flies after eclosion and testes were removed from one to three days old flies in PBS buffer using sharp tweezers. 


\section{P-element reversion screen}

P-element reversion [21] was carried out by crossing female $w^{1118}$; $d m f r n^{S H 115}$ virgins with $w^{1118} ; w g / C y O ; \Delta 2-3$ $\mathrm{Sb} / \mathrm{TM} 6 \mathrm{c} \mathrm{Tb}$ male flies that carry the immobilized transposase source $\Delta 2-3$ [51]. F1 males of the genotype $w^{1118}$; $\mathrm{CyO} /+; \Delta 2-3 \mathrm{Sb} / \mathrm{dmfrn}^{\mathrm{SH} 115}$ (mosaic expression of white) were crossed to virgin $w^{1118}$; Vno/TM6c Sb, Tb female flies. Single white eyed male F2 of the genotype $w^{1118 ;}+/$ +; ?/Vno were crossed to virgin $w^{1118 ;}$ Vno/TM6c Sb, Tb. Finally, F3 $w^{1118}$; ?/TM6c Sb, Tb siblings were used to establish lines. These were then analyzed by PCR for the absence of $P\{l a c W\} S H 115^{l(3) S H 115}$.

\section{Fertility assay}

Single 3-4 days old male or female flies, collected from flies reared on indicated food sources, were mated with $2-3$ virgin $w^{1118}$ female or 2 male $w^{1118}$ flies reared on normal food, respectively. After 5-7 days of mating, the fraction of fertile flies was determined by the presence of larvae.

\section{Transgenic dmfrnvenus flies}

The genomic region of $d m f r n$ was subcloned in several steps to generate a genomic construct, tagged by a C-terminal fusion with the coding sequence of the fluorescent protein venus. For all PCR reactions, Phusion high fidelity polymerase (Finnzymes) was used with either $w^{1118}$ genomic DNA, or plasmid pHWV (Carnegie Drosophila Gateway Collection) as a template for $d m f r n$ or venus respectively. Restriction digestions were carried out with enzymes from New England Biolabs. Arctic shrimp alkaline phosphatase and the Rapid DNA Ligation Kit were obtained from ROCHE Applied Science.

Using primers 5' ACT AGT CTA GGA GCA GCA GGC CCA C 3' (introducing SpeI and a stop codon in the first exon of Gp93) and 5' AAA AAT CGA TAA AAG CTA GCC GTG CTG AAG CCC CGC TCG 3' (introducing NheI and ClaI) the region from the first exon of GP93 to the end of the coding sequence of $d m f r n$, omitting the stop codon, was subcloned into pCR-XL-TOPO (Invitrogen) by TOPO cloning (Invitrogen). Using primers $5^{\prime}$ AAA AGC TAG CAT GGT GAG CAA GGG CGA G 3' (introducing a NheI site) and 5' AAA AAT CGA TTC ACG TGG ACC GGT GCT T 3' (introducing a ClaI site), the coding sequence of the fluorescent protein venus was PCR amplified, and cloned in frame behind the coding sequence of dmfrn using restriction sites NheI and ClaI. Next, the 3'UTR of dmfrn was PCR amplified, using primers 5' AAA AAT CGA TAC GTA GGC GTC GCC GGT GG 3' (introducing $\mathrm{ClaI}$ ) and 5' AAA AGG TAC CCG GAA ACA ATA AAA GGC AAT TGT TG 3' (introducing a KpnI site) and was cloned behind the coding sequence of venus, using the introduced ClaI site of the previous step and a KpnI site within the plasmid. Cloned fragments were sequenced after each step. Finally, the genomic construct was cloned into pCasper4 [52] using restriction sites SpeI and KpnI and the resulting plasmid was sent for co-injection with plasmid $\mathrm{p} \Delta 2-3$ into $w^{1118}$ embryos for generation of transgenic flies at the Department of Developmental Biology, Wenner-Gren Institute, Stockholm University. Transgenic $d m f r n^{\text {venus }}$ flies were verified by PCR and outcrossed for four generations to $w^{1118}$ flies.

\section{RT-RT PCR}

RNA was extracted from tissues prepared from 2 to 3 days old virgin male $w^{1118}$ adult flies, raised at room temperature on potato food. Dissections were carried out in PBS buffer on a wax plate on ice and tissues were dissolved directly in $400 \mu \mathrm{L} \mathrm{1 \%} 2$-mercaptoethanol RLT buffer from the RNeasy Kit (QIAGEN). Heads and thoraxes were ripped open before lysis and disrupted using a micro pestle. Tissues from 20 flies were pooled per experiment and after passing lysates through QIAShredder columns (QIAGEN), RNA was purified from $350 \mu \mathrm{L}$ flow through using the RNeasy Kit (QIAGEN). Integrity of RNA was analyzed spectrophotometrically and by agarose gelectrophoresis. cDNA was synthesized from 350 ng total RNA using the QuantiTect reverse transcription kit (QIAGEN) performing the gDNA wipeout reaction to remove genomic DNA contaminations. The cDNA synthesis reaction was also performed without QScript RTase and used as a negative control PCR reaction to test for gDNA contamination. The QuantiTect SYBR green kit (QIAGEN) was used for RT-RT PCR in a RotorGene 3000 (Corbett Research) thermocycler using primers for cDNAs of genes Rp49 [53], dmfrn, Fer1HCH, Fer2LCH [13], Fer3HCH (CG4349), forward 5'- GAA GGC ATC CCA CCA GTA TC-3', reverse 5'- GGC TGT GGT ACA CTG CTC AA-3' and frataxin homolog (CG8971) forward 5'- ACA AGC ACA GTG GTC AGT CG-3', reverse 5'- TAC AGT AGG GCA GGC GTA GG -3'.

\section{X-gal Staining}

Testes were fixed in 3.7\% (w/v) formaldehyde in PBS for 15-30 $\mathrm{min}$ at RT. Fixed testes were washed twice for 10 min with PBS, permeabilized for 20 min with PBST (PBS, $0.3 \%(\mathrm{v} / \mathrm{v})$ Triton $\mathrm{X}-100)$ and then stained with staining solution (10\% (w/v) 5-Bromo-4-chloro-3-indolyl $\beta$-Dgalactoside $(\mathrm{X}$-gal) in dimethyl sulfoxide was added to a final concentration of $0.2 \%(\mathrm{w} / \mathrm{v})$ to preheated staining buffer (10 mM phosphate buffer pH 7.2, $150 \mathrm{mM} \mathrm{NaCl}, 1$ $\mathrm{mM} \mathrm{MgCl}, 3 \mathrm{mM} \mathrm{K}_{4}\left(\mathrm{Fe}(\mathrm{II})(\mathrm{CN})_{6}, \mathrm{~K}_{3}\left(\mathrm{Fe}(\mathrm{III})(\mathrm{CN})_{6}, 0.3 \%\right.\right.$ (v/v) Triton $\mathrm{X}-100)$ ) at $37^{\circ} \mathrm{C}$ until colorization was visible. Testes were first rinsed with $\mathrm{NaCl}-\mathrm{T}(0.7 \%(\mathrm{w} / \mathrm{v}) \mathrm{NaCl}$, $0.3 \%(\mathrm{v} / \mathrm{v})$ Triton X-100) then with water, mounted in PBS and imaged by conventional light microscopy. 
Testes preparation, testes squashes and fluorescence staining of testes and fluorescence microscopy

Testes of 1-3 days old virgin males were dissected out in a drop of PBS buffer using fine forceps (\#5, FineScience Tools), transferred to a small drop of PBS on a microscope slide and carefully ripped open to release their content under the weight of a coverslip. Excess buffer was removed using a paper cloth, while observing the squash under phase contrast settings on a Leica TSC-SP microscope.

For fluorescence microscopy, testes were collected in Schneider Drosophila Medium (SDM) and stained with $100 \mathrm{nM}$ MitoTracker Deep Red $633 \mathrm{~nm}$ (Invitrogen) in SDM for 2 hours. Testes were washed twice with PBS, fixed with $3.7 \%(\mathrm{w} / \mathrm{v})$ formaldehyde in PBS, washed twice with PBS and permeabilized with $0.3 \%(\mathrm{v} / \mathrm{v})$ Triton X-100 in PBS. After washing twice with PBS, testes were stained with Rhodamine Phalloidin (diluted 1:1000 in PBS; Invitrogen), washed three times with PBS and mounted in VECTASHIELD Mounting Medium with or without DAPI (Vector Labs). Specimens were either imaged using conventional fluorescent microscopy on a Leica TSC-SP or confocal laser scanning microscopy on a Leica TCSNT or a Zeiss LSM510 confocal microscope.

\section{Transmission electron microscopy}

Glutharaldehyde fixed testes were dehydrated, embedded, sectioned and stained following standard procedures at the Biological Structure Analysis Facility, Uppsala University, Sweden. Sections were imaged using a Zeiss Supra35VP electron microscope.

\section{Additional material}

Additional file 1 Confirmation of fly lines used in the study, sper-

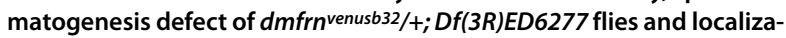
tion of ferritin during spermatogenesis. Contains text, a table with primers and images.

\section{Authors' contributions}

CM designed and performed all experiments, interpreted the results, drafted and wrote the manuscript. MIL discussed the experiments and results and critically read the manuscript. Both authors read and approved the final manuscript.

\section{Acknowledgements}

We are indebted to Mitchell S. Dushay (Deptartment of Biology, Illinois Institute of Technology, College of Science and Letters, Chicago, USA) who taught us to work with Drosophila during his employment at the Department of Comparative Physiology, Uppsala University, Sweden. We are grateful for the communication with Karen G. Hales (Davidson College, N.C., USA), who helped us with the testis squashes and critically read early versions of the manuscript. Funding

This work was supported by grants from the Wenner-Gren Foundations, the Carl Tryggers Stiftelse (grant number CTS08:226), the Magnus Bergvall's Foundation, and by the Swedish Research Council (grant number 621-2008-3669) to MIL. The work was also supported by a grant from the Swedish Research Council (grant number 621-2006-4658) to Kenneth Söderhäll (Department of Comparative Physiology, Evolutionary Biology Centre, Uppsala University, Uppsala, Sweden).

\author{
Author Details \\ Comparative Physiology, Uppsala University, Norbyvägen 18A, 75236 Uppsala, \\ Sweden
}

Received: 11 January 2010 Accepted: 21 June 2010

Published: 21 June 2010

\section{References}

1. Andrews NC: Forging a field: the golden age of iron biology. Blood 2008, 112:219-230.

2. Dunn LL, Rahmanto YS, Richardson DR: Iron uptake and metabolism in the new millennium. Trends Cell Biol 2007, 17:93-100

3. Lill $R$, Mühlenhoff $U$ : Maturation of iron-sulfur proteins in eukaryotes: mechanisms, connected processes, and diseases. Annu Rev Biochem 2008, 77:669-700.

4. Rouault TA, Tong WH: Iron-sulfur cluster biogenesis and human disease. Trends Genet 2008, 24:398-407

5. Wiesenberger G, Link TA, von Ahsen U, Waldherr M, Schweyen RJ: MRS3 and MRS4, two suppressors of mtRNA splicing defects in yeast, are new members of the mitochondrial carrier family. J Mol Biol 1991, 217:23-37.

6. Froschauer EM, Schweyen RJ, Wiesenberger G: The yeast mitochondrial carrier proteins Mrs3p/Mrs4p mediate iron transport across the inner mitochondrial membrane. Biochim Biophys Acta 2009, 1788:1044-1050.

7. Roganti T: Deletion of the mitochondrial carrier genes MRS3 and MRS4 suppresses mitochondrial iron accumulation in a yeast frataxindeficient strain. J Biol Chem 2002, 277:24475-24483.

8. Zhang Y, Lyver ER, Knight SA, Lesuisse E, Dancis A: Frataxin and mitochondrial carrier proteins, Mrs3p and Mrs4p, cooperate in providing iron for heme synthesis. J Biol Chem 2005, 280:19794-19807.

9. Zhang Y, Lyver ER, Knight SA, Pain D, Lesuisse E, Dancis A: Mrs3p, Mrs4p, and frataxin provide iron for Fe-S cluster synthesis in mitochondria. $J$ Biol Chem 2006, 281:22493-22502.

10. Mühlenhoff U, Stadler JA, Richhardt N, Seubert A, Eickhorst T, Schweyen RJ, Lill R, Wiesenberger G: A specific role of the yeast mitochondrial carriers MRS3/4p in mitochondrial iron acquisition under iron-limiting conditions. J Biol Chem 2003, 278:40612-40620.

11. Shaw GC, Cope JJ, Li L, Corson K, Hersey C, Ackermann GE, Gwynn B, Lambert AJ, Wingert RA, Traver D, Trede NS, Barut BA, Zhou Y, Minet E, Donovan A, Brownlie A, Balzan R, Weiss MJ, Peters LL, Kaplan J, Zon LI, Paw $\mathrm{BH}$ : Mitoferrin is essential for erythroid iron assimilation. Nature 2006, 440:96-100

12. Paradkar PN, Zumbrennen KB, Paw BH, Ward DM, Kaplan J: Regulation of mitochondrial iron import through differential turnover of mitoferrin 1 and mitoferrin 2. Mol Cell Biol 2009, 29:1007-1016.

13. Metzendorf C, Wu W, Lind MI: Overexpression of Drosophila mitoferrin in I(2)mbn cells results in dysregulation of Fer $1 \mathrm{HCH}$ expression. Biochem J 2009, 421:463-471.

14. Arama E, Bader M, Srivastava M, Bergmann A, Steller H: The two Drosophila cytochrome $C$ proteins can function in both respiration and caspase activation. EMBO J 2006, 25:232-243.

15. Hales KG, Fuller MT: Developmentally regulated mitochondrial fusion mediated by a conserved, novel, predicted GTPase. Cell 1997 90:121-129.

16. Hannah-Alava A: The premeiotic stages of spermatogenesis. Adv Genet 1965, 13:157-226.

17. Bairati A: [The structure and ultrastructure of the male genital apparatus of the Drosophila melanogaster Meig. 1. The testis]. Z Zellforsch Mikrosk Anat 1967, 76:56-99.

18. Tokuyasu KT, Peacock WJ, Hardy RW: Dynamics of spermiogenesis in Drosophila melanogaster. I. Individualization process. ZZellforsch Mikrosk Anat 1972, 124:479-506.

19. Oh SW, Kingsley T, Shin HH, Zheng Z, Chen HW, Chen X, Wang H, Ruan P, Moody M, Hou SX: A P-element insertion screen identified mutations in 455 novel essential genes in Drosophila. Genetics 2003, 163:195-201.

20. Staudt N, Molitor A, Somogyi K, Mata J, Curado S, Eulenberg K, Meise M, Siegmund T, Hader T, Hilfiker A, Bronner G, Ephrussi A, Rorth P, Cohen SM, Fellert S, Chung HR, Piepenburg O, Schafer U, Jackle H, Vorbruggen G: Gain-of-function screen for genes that affect Drosophila muscle pattern formation. PLoS Genet 2005, 1:e55.

21. Cooley L, Kelley R, Spradling A: Insertional mutagenesis of the Drosophila genome with single P elements. Science 1988, 239:1121-1128. 
22. Chintapalli VR, Wang J, Dow JA: Using FlyAtlas to identify better Drosophila melanogaster models of human disease. Nat Genet 2007, 39:715-720

23. White-Cooper $\mathrm{H}$ : Spermatogenesis: analysis of meiosis and morphogenesis. Methods Mol Biol 2004, 247:45-75.

24. Castrillon DH, Gonczy P, Alexander S, Rawson R, Eberhart CG, Viswanathan S, DiNardo S, Wasserman SA: Toward a molecular genetic analysis of spermatogenesis in Drosophila melanogaster: characterization of malesterile mutants generated by single P element mutagenesis. Genetics 1993, 135:489-505.

25. Tokuyasu KT: Dynamics of spermiogenesis in Drosophila melanogaster. 3. Relation between axoneme and mitochondrial derivatives. Exp Cell Res 1974, 84:239-250.

26. Levi S, Corsi B, Bosisio M, Invernizzi R, Volz A, Sanford D, Arosio P, Drysdale $\mathrm{J}$ : A human mitochondrial ferritin encoded by an intronless gene. J Biol Chem 2001, 276:24437-24440.

27. Santambrogio P, Biasiotto G, Sanvito F, Olivieri S, Arosio P, Levi S: Mitochondrial ferritin expression in adult mouse tissues. J Histochem Cytochem 2007, 55:1129-1137

28. Missirlis F, Holmberg S, Georgieva T, Dunkov BC, Rouault TA, Law JH: Characterization of mitochondrial ferritin in Drosophila. Proc Natl Acad SciUSA 2006, 103:5893-5898.

29. Nichol H, Law JH, Winzerling JJ: Iron metabolism in insects. Annu Rev Entomol 2002, 47:535-559

30. Missirlis F, Kosmidis S, Brody T, Mavrakis M, Holmberg S, Odenwald WF Skoulakis EM, Rouault TA: Homeostatic mechanisms for iron storage revealed by genetic manipulations and live imaging of Drosophila ferritin. Genetics 2007, 177:89-100

31. Bier E, Vaessin H, Shepherd S, Lee K, McCall K, Barbel S, Ackerman L, Carretto R, Uemura T, Grell E: Searching for pattern and mutation in the Drosophila genome with a P-lacZ vector. Genes Dev 1989, 3:1273-1287.

32. Li FY, Leibiger B, Leibiger I, Larsson C: Characterization of a putative murine mitochondrial transporter homology of hMRS3/4. Mamm Genome 2002, 13:20-23.

33. Koutnikova H, Campuzano V, Foury F, Dolle P, Cazzalini O, Koenig M: Studies of human, mouse and yeast homologues indicate a mitochondrial function for frataxin. Nat Genet 1997, 16:345-351.

34. Orlando C, Caldini AL, Barni T, Wood WG, Strasburger CJ, Natali A, Maver A, Forti G, Serio M: Ceruloplasmin and transferrin in human seminal plasma: are they an index of seminiferous tubular function? Fertil Steril 1985, 43:290-294.

35. Sylvester SR, Griswold MD: Localization of transferrin and transferrin receptors in rat testes. Biol Reprod 1984, 31:195-203.

36. Skinner MK, Griswold MD: Sertoli cells synthesize and secrete transferrin-like protein. J Biol Chem 1980, 255:9523-9525.

37. Sylvester SR, Griswold MD: The testicular iron shuttle: a "nurse" function of the Sertoli cells. J Androl 1994, 15:381-385.

38. Cavadini P, Biasiotto G, Poli M, Levi S, Verardi R, Zanella I, Derosas M, Ingrassia R, Corrado M, Arosio P: RNA silencing of the mitochondrial ABCB7 transporter in HeLa cells causes an iron-deficient phenotype with mitochondrial iron overload. Blood 2007, 109:3552-3559.

39. Rutherford JC, Ojeda L, Balk J, Mühlenhoff U, Lill R, Winge DR: Activation of the iron regulon by the yeast Aft1/Aft2 transcription factors depends on mitochondrial but not cytosolic iron-sulfur protein biogenesis. J Biol Chem 2005, 280:10135-10140.

40. Pandolfo M: Frataxin deficiency and mitochondrial dysfunction. Mitochondrion 2002, 2:87-93.

41. Li L, Kaplan J: A mitochondrial-vacuolar signaling pathway in yeast that affects iron and copper metabolism. J Biol Chem 2004, 279:33653-33661

42. Kamboj VP, Kar AB: Effect of iron salts on the genital organs and fertility of male rats. Acta Biol Med Ger 1964, 13:928-945.

43. Kamboj VP, KAR AB: Antitesticular effect of metallic and rare earth salts. J Reprod Fertil 1964, 7:21-8.

44. Detmer SA, Chan DC: Functions and dysfunctions of mitochondrial dynamics. Nat Rev Mol Cell Biol 2007, 8:870-879.

45. Arama E, Agapite J, Steller H: Caspase activity and a specific cytochrome C are required for sperm differentiation in Drosophila. Dev Cell 2003, 4:687-697.

46. Nichol H, Locke M: Secreted ferritin subunits are of two kinds in insects molecular cloning of cDNAs encoding two major subunits of secreted ferritin from Calpodes ethlius. Insect Biochem Mol Biol 1999, 29:999-1013.
47. Steiger D, Fetchko M, Vardanyan A, Atanesyan L, Steiner K, Turski ML, Thiele DJ, Georgiev O, Schaffner W: The Drosophila copper transporter Ctr1C functions in male fertility. J Biol Chem 2010, 285:17089-97.

48. White-Cooper $\mathrm{H}$ : Studying how flies make sperm--investigating gene function in Drosophila testes. Mol Cell Endocrinol 2009, 306:66-74.

49. Ryder E, Blows F, Ashburner M, Bautista-Llacer R, Coulson D, Drummond J, Webster J, Gubb D, Gunton N, Johnson G, O'Kane CJ, Huen D, Sharma P, Asztalos Z, Baisch H, Schulze J, Kube M, Kittlaus K, Reuter G, Maroy P, Szidonya J, Rasmuson-Lestander A, Ekstrom K, Dickson B, Hugentobler C, Stocker H, Hafen E, Lepesant JA, Pflugfelder G, Heisenberg M, Mechler B, Serras F, Corominas M, Schneuwly S, Preat T, Roote J, Russell S: The DrosDel collection: a set of P-element insertions for generating custom chromosomal aberrations in Drosophila melanogaster. Genetics 2004, 167:797-813.

50. Ryder E, Ashburner M, Bautista-Llacer R, Drummond J, Webster J, Johnson G, Morley T, Chan YS, Blows F, Coulson D, Reuter G, Baisch H, Apelt C, Kauk A, Rudolph T, Kube M, Klimm M, Nickel C, Szidonya J, Maroy P, Pal M, Rasmuson-Lestander A, Ekstrom K, Stocker H, Hugentobler C, Hafen E, Gubb D, Pflugfelder G, Dorner C, Mechler B, Schenkel H, Marhold J, Serras F, Corominas M, Punset A, Roote J, Russell S: The DrosDel deletion collection: a Drosophila genomewide chromosomal deficiency resource. Genetics 2007, 177:615-629.

51. Robertson HM, Preston CR, Phillis RW, Johnson-Schlitz DM, Benz WK, Engels WR: A stable genomic source of $P$ element transposase in Drosophila melanogaster. Genetics 1988, 118:461-470.

52. Thummel CS, Pierrotta V: Technical notes: new pCaSpeR P-element vectors. Drosophila Information Service 1992, 71:150.

53. Girardot F, Monnier $V$, Tricoire H: Genome wide analysis of common and specific stress responses in adult Drosophila melanogaster. BMC Genomics 2004, 5:74.

doi: $10.1186 / 1471-213 X-10-68$

Cite this article as: Metzendorf and Lind, Drosophila mitoferrin is essential for male fertility: evidence for a role of mitochondrial iron metabolism during spermatogenesis BMC Developmental Biology 2010, 10:68

\section{Submit your next manuscript to BioMed Central and take full advantage of:}

- Convenient online submission

- Thorough peer review

- No space constraints or color figure charges

- Immediate publication on acceptance

- Inclusion in PubMed, CAS, Scopus and Google Scholar

- Research which is freely available for redistribution 